\title{
Stabilization of ribozyme-like cis-noncoding rRNAs induces apoptotic and nonapoptotic death in lung cells
}

\author{
M Gee ${ }^{1}$, Y Gu$^{1}$, JR Fields ${ }^{1}$ and Y-H Shiao*,1
}

Bidirectional non-protein-coding RNAs are ubiquitously transcribed from the genome. Convergent sense and antisense transcripts may regulate each other. Here, we examined the convergent cis-noncoding rRNAs (nc-rRNAs) in A5 and E9 lung cancer models. Sense nc-rRNAs extending from $r D N A$ intergenic region to internal transcribed spacer of around $10 \mathrm{~kb}$ in length were identified. nc-rRNAs in sense direction exhibited in vitro characteristics of ribozymes, namely, degradation upon incubation with $\mathrm{MgCl}_{2}$ and stabilization by complementary oligonucleotides. Detection of endogenous cleavage-ligation products carrying internal deletion of hundreds to thousands nucleotides by massively parallel sequencing confirmed the catalytic properties. Transfection of oligonucleotides pairing with antisense nc-rRNAs stabilized both target and complementary transcripts, perturbed $r R N A$ biogenesis, and induced massive cell death via apoptotic and/or nonapoptotic mechanisms depending on cell type and treatment. Oligonucleotides targeting cellular sense transcripts are less responsive. Spontaneously detached cells, though rare, also showed accumulation of nc-rRNAs and perturbation of $r R N A$ biogenesis. Direct participation of nc-rRNAs in apoptotic and nonapoptotic death was demonstrated by transfection of synthetic nc-rRNAs encompassing the rDNA promoter. In sum, convergent cis-nc-rRNAs follow a feed-forward mechanism to regulate each other and $r R N A$ biogenesis. This opens an opportunity to disrupt $r R N A$ biogenesis, commonly upregulated in cancers, via inhibition of ribozyme-like activities in nc-rRNAs. Cell Death and Disease (2012) 3, e281; doi:10.1038/cddis.2012.19; published online 15 March 2012

Subject Category: Cancer

Noncoding RNAs are major components of mammalian transcriptomes $^{1,2}$ and are transcribed in sense and/or antisense directions from 60 to $70 \%$ of genomes. ${ }^{3,4}$ Among tens of thousands of transcription clusters examined, over $20 \%$ in humans and about $72 \%$ in mice are expressed as sense-antisense pairs. ${ }^{5-7}$ The levels of sense and antisense noncoding RNA are either concordant, represented by concurrent increase or decrease, or discordant, also described as reciprocal regulation. ${ }^{7,8}$ Antisense transcripts, mostly noncoding for proteins, are known to regulate transcriptions of many protein-coding genes in the sense orientation, including those involved in development, immune cell maturation, neurodegenerative diseases, and cancers. ${ }^{8}$ Transcriptional regulation is also carried out by upstream intergenic cis-noncoding RNAs overlapping with downstream coding transcripts, such as for the DHFR gene in humans ${ }^{9}$ and for the Ser3 gene in yeasts. ${ }^{10}$ Noncoding RNAs are detectable using common techniques, including sensitive reverse transcription-polymerase chain reaction (RT-PCR) and high-throughput massively parallel DNA sequencing. However, overlapping of the cis-noncoding and primary transcripts poses a big challenge to identify full-length cis-RNA species for functional characterization.

Intergenic noncoding rRNA (nc-rRNA) transcripts have been observed in rodents and humans. ${ }^{11-13} A$ segment of the cis-nc-rRNA has been shown to regulate transcription of the primary $r R N A$ in mouse fibroblast cells. ${ }^{14}$ The primary $r R N A$ transcript, precursor of the 18S, 5.8S, and $28 S$ rRNAs, undergoes serial steps of processing that begins with excision of the leader sequence from the $5^{\prime}$-external transcribed spacer at about +650 nucleotide downstream of the transcription start site in mice. ${ }^{15,16}$ Proliferating cells exhibit high levels of ribosome production as well as increased rRNA biogenesis, including transcription and subsequent processing, that are orchestrated by many oncogenes and tumor-suppressor genes. ${ }^{17,18}$ The effects of sense-antisense nc-rRNAs on $r R N A$ biogenesis and cell phenotype are still unclear. In this study, we obtained mouse strain-specific rDNA sequence and observed that both sense and antisense nc-rRNAs were extensively expressed in corresponding A5 and E9 mouse lung cells, commonly used cancer models. ${ }^{19}$ A protocol was developed to determine full-length sequences of the sense nc-rRNAs overlapping with the primary $r R N A$. Catalytic properties of nc-rRNAs were identified. The roles of sense and antisense cis-nc-rRNAs in $r R N A$ biogenesis, as well as in cell growth and death, were examined. The potential of targeting nc-rRNAs for anticancer treatment is also discussed.

\section{Results}

Detection of extensive bidirectional cis-nc-rRNAs. More than $14.4 \mathrm{kbp}$ of the $r D N A$ covering most of the transcribed

\footnotetext{
${ }^{1}$ Laboratory of Comparative Carcinogenesis, National Cancer Institute at Frederick, Frederick, MD 21702, USA

${ }^{*}$ Corresponding author: Y-H Shiao, Laboratory of Comparative Carcinogenesis, National Cancer Institute at Frederick, Building 538, Room 205, NCl-Frederick, $\mathrm{NIH}$, West 7th Street, Frederick, MD 21702, USA. Tel: + 301846 1246; Fax: + 301846 5946; E-mail: shiaoy@mail.nih.gov

Keywords: rRNA; noncoding RNA; ribozyme; antisense; apoptosis

Abbreviations: RT-PCR, reverse transcription-polymerase chain reaction; nc-rRNA, noncoding rRNA; bp, base pair; LNA, locked nucleic acid; DEPC, diethyl pyrocarbonate

Received 19.7.11; revised 27.1.12; accepted 27.1.12; Edited by A Stephanou
} 
region for primary $r R N A$ in A5 lung cells of BALB/c mouse background were sequenced (GenBank GU372691) using primers selected from our assembled C57BL6 mouse sequence (see Materials and Methods). The same primers were used to detect sense as well as antisense nc-rRNAs in extensive regions of the $r D N A$ gene (Figure 1). Their identities were confirmed by dideoxy DNA sequencing. At least three fragments of antisense nc-rRNAs were observed and the most upstream transcription start site was located outside the $28 \mathrm{~S}$ region. Sense nc-rRNAs were transcribed several hundreds to thousands of nucleotides upstream from the primary transcription start site. The downstream regions of the sense nc-rRNAs overlapped with the primary transcript and determination of their $3^{\prime}$ sequences required direct separation of the two types of transcripts. The extensive bidirectional cis-nc-rRNAs were also detected in the E9 lung cell line of the same mouse background.

Identification of long sense nc-rRNAs. A streptavidincoated magnetic capture-hybridization method was initially applied to isolate sense nc-rRNAs from mouse lung cells using only one biotin-tagged probe specific to an upstream region of the $r R N A$. Initially, recovered sense nc-rRNAs were hardly detectable using RT-PCR. Poor binding by a single probe and RNA degradation were suspected and tested by including two additional probes along with 30 complementary oligonucleotides across the rDNA sequence spaced about 500 nucleotides apart. Hundred- to thousand-fold enrichments were observed, as indicated by the increase of the ratio of sense nc-rRNAs to the $18 S$ rRNA and Gapdh reference transcripts (Figure 2a). Nevertheless, the signal for the two references remained extremely high, suggesting nonspecific binding of these reference transcripts to magnetic microparticles. Such undesired binding of nontarget RNAs to excessive streptavidin epitopes was quenched by free biotin and further 3-5-fold enrichments of sense nc-rRNAs were achieved by addition of $0.3 \mu \mathrm{M}$ biotin in the wash solution (Figure 2a). 10-fold higher or lower biotin concentration attenuated the enrichment.

To determine the transcription start and end sites, the enriched sense nc-rRNAs were circularized with T4 RNA ligase and an intergenic primer was designed specifically to reverse-transcribe the circular RNAs across the joined $5^{\prime}$ - and $3^{\prime}$-termini. In all, 24 serial forward primers (Supplementary Table S2) about 500 nucleotides apart across the rDNA sequence were used along with the same reverse primer in an attempt to amplify products containing the $5^{\prime}$ - and $3^{\prime}$-termini from circularized nc-rRNAs. Sequence alignment using Basic Local Alignment Search Tool 2 algorithm (http:// blast.ncbi.nlm.nih.gov/Blast.cgi) identified two transcripts, approximately $10 \mathrm{~kb}$ in length, starting at about 300 nucleotides upstream of the transcription start site and ending in the ITS2 region (Figure $2 \mathrm{~b}$ ). The products were repeatedly detected in separate assays using the combination of -69 / -50 reverse and +7020 forward primers. It is noteworthy that the two transcripts of slight length variation are also differentiable by a single-nucleotide polymorphism at the -104 nucleotide.

Ribozyme-like characteristics of sense nc-RNAs. Observation of the enhanced sense nc-rRNA recovery with the presence of 30 oligonucleotides during magnetic capturehybridization steps suggests that sense nc-rRNAs may carry self-catalytic potential. To test this, total RNA was incubated with a common ribozyme cofactor $\mathrm{MgCl}_{2}$. If sense nc-rRNAs possess self-catalytic properties, $\mathrm{MgCl}_{2}$ would promote RNA catalysis and complementary oligonucleotides specifically to the transcripts would prevent the catalysis. Indeed, the signal

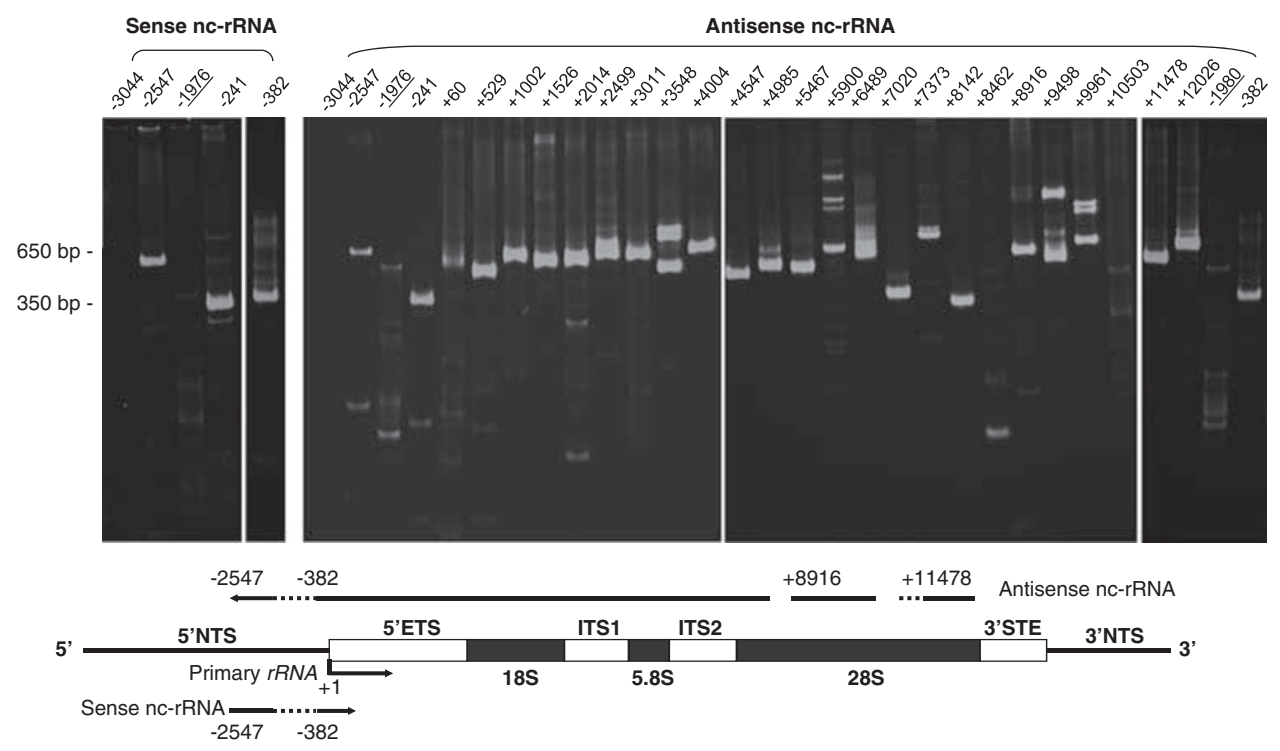

Figure 1 Extensive sense and antisense nc-rRNA transcripts within the rDNA locus. The gel lanes show amplified transcripts by RT-PCR and their locations in the rRNA are identified by their $5^{\prime}$-terminal nucleotides. Those lanes labeled with underlined nucleotide identifiers, also presented as dotted lines in the bottom figure, represent regions that cannot be determined because amplification was unsuccessful even from DNA template. NTS, nontranscribed spacer; ETS, external transcribed spacer; ITS, internal transcribed spacer. See Supplementary Table 1 for the corresponding nucleotide locations 
a
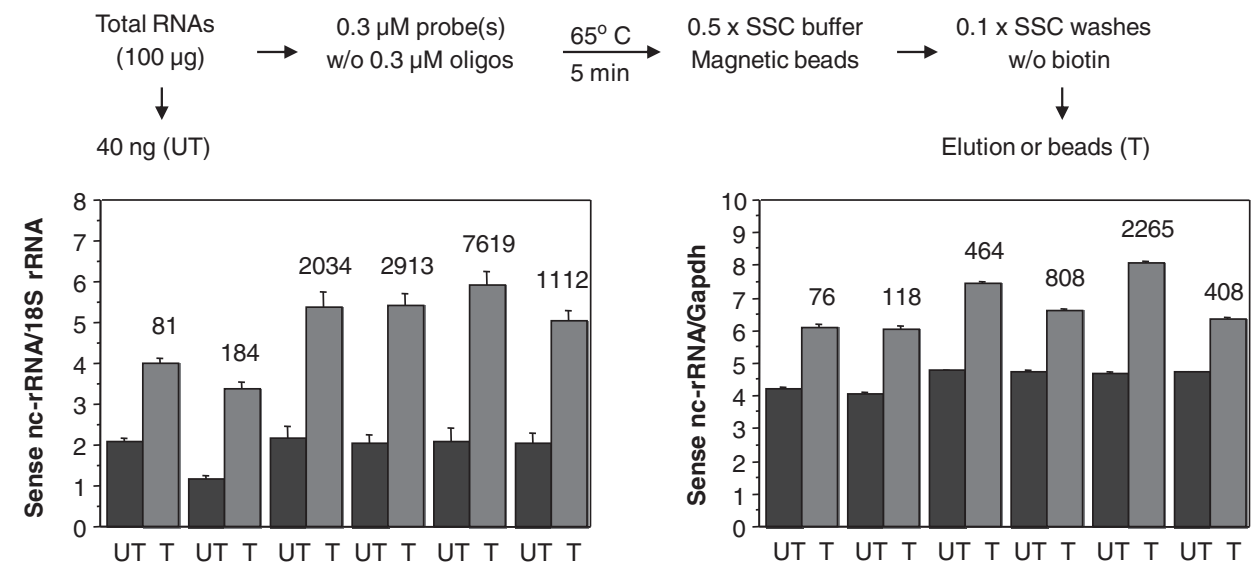

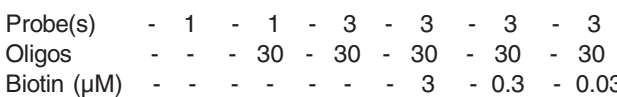

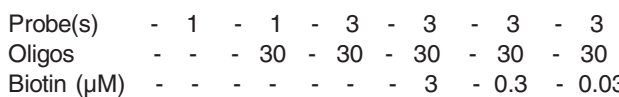

b
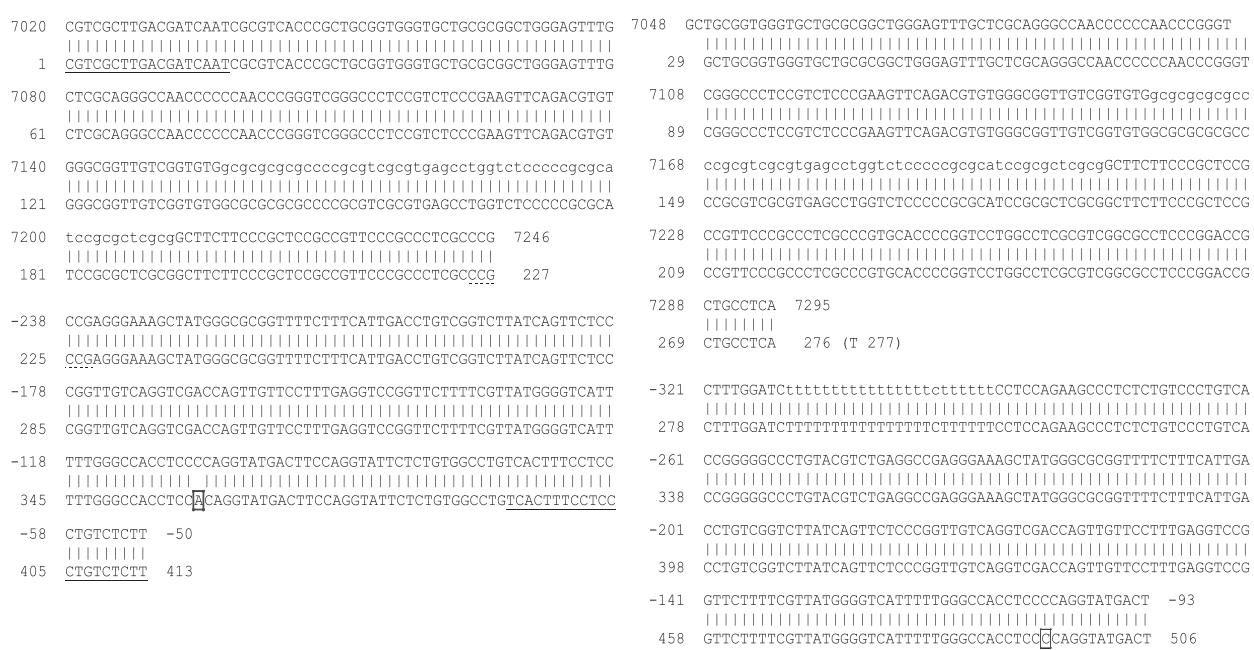

Figure 2 Enrichment of sense nc-rRNAs and identification of $5^{\prime}$ - and $3^{\prime}$-termini of sense nc-rRNAs. (a) The ratio of RT-PCR intensity between sense nc-rRNAs and the reference transcript, either the 18S rRNA or Gapdh, was used to calculate the fold enrichment (values inside the histogram) after magnetic purification treatment (T), compared with an aliquot of untreated crude RNA before the purification (UT). All enrichments were statistically significant. The linear scale in Y-axis represents transformed values using $\log \left(\right.$ original ratio $\left.\times 10^{7}\right)$. Error bars denote $\pm \mathrm{S}$.E. (b) Alignment of two amplified products (bottom sequences at left and right panels) to the BALB/c rDNA sequence (GenBank GU372691, top sequences, number in reference to transcription start site). The dotted/underlined CCG trinucleotides on the left panel cannot be assigned unambiguously to either upstream or downstream sequences. A differential single-nucleotide polymorphism is marked by the rectangular box in these two sequences. The T nucleotide at 277 on the right panel does not align with the $r D N A$ sequence

of sense nc-rRNAs to the reference $18 S$ rRNA of $600 \mathrm{bp}$ in size was significantly reduced after incubation with $\mathrm{MgCl}_{2}$ and pretreatment with 30 complementary oligonucleotides alleviated the reduction (Figure 3a). The Gapdh transcript was not protected by the same rRNA- or Gapdh-selective oligonucleotides (Figures $3 \mathrm{~b}$ and $\mathrm{c}$ ), demonstrating noncatalytic property of mature Gapdh and the specificity of oligonucleotide-mediated attenuation to the putative ribozyme activity in sense nc-rRNAs. Indistinguishable stability between the long 600-bp and the short 65-bp (rRNA65) regions in the $18 S$ rRNA (Figure 3d) predicts that $\mathrm{MgCl}_{2}$ is unlikely to alter the stability of the 600-bp $r R N A$. This strengthens the observation that changes of sense nc-rRNA/18S rRNA ratios were due to RNA catalysis of sense nc-rRNAs, not the increase of the 600-bp $18 S$ rRNA stability.

Identification of catalytic sense nc-rRNAs in mouse lung cells. Finding of the above ribozyme-like characteristics of sense nc-rRNAs prompted us to search for endogenous cleavage-ligation ribozyme products using the massively parallel deep sequencing. The products were first amplified using a single upstream primer in combination with multiple downstream primers (see Materials and Methods). About 22600 sequence reads of an average of $73 \mathrm{bp}$ in length were collected from 26 pooled PCR products. A total of 41 sequences aligned to both upstream and downstream regions of the BALB/c rDNA gene (GenBank GU372691) but 
showed loss of internal portions that generated gaps of hundreds to thousands of nucleotides (Table 1). The frequent cleavage-ligation sites occurred between the -226 to -44 nucleotides in the upstream and two downstream hot-spot
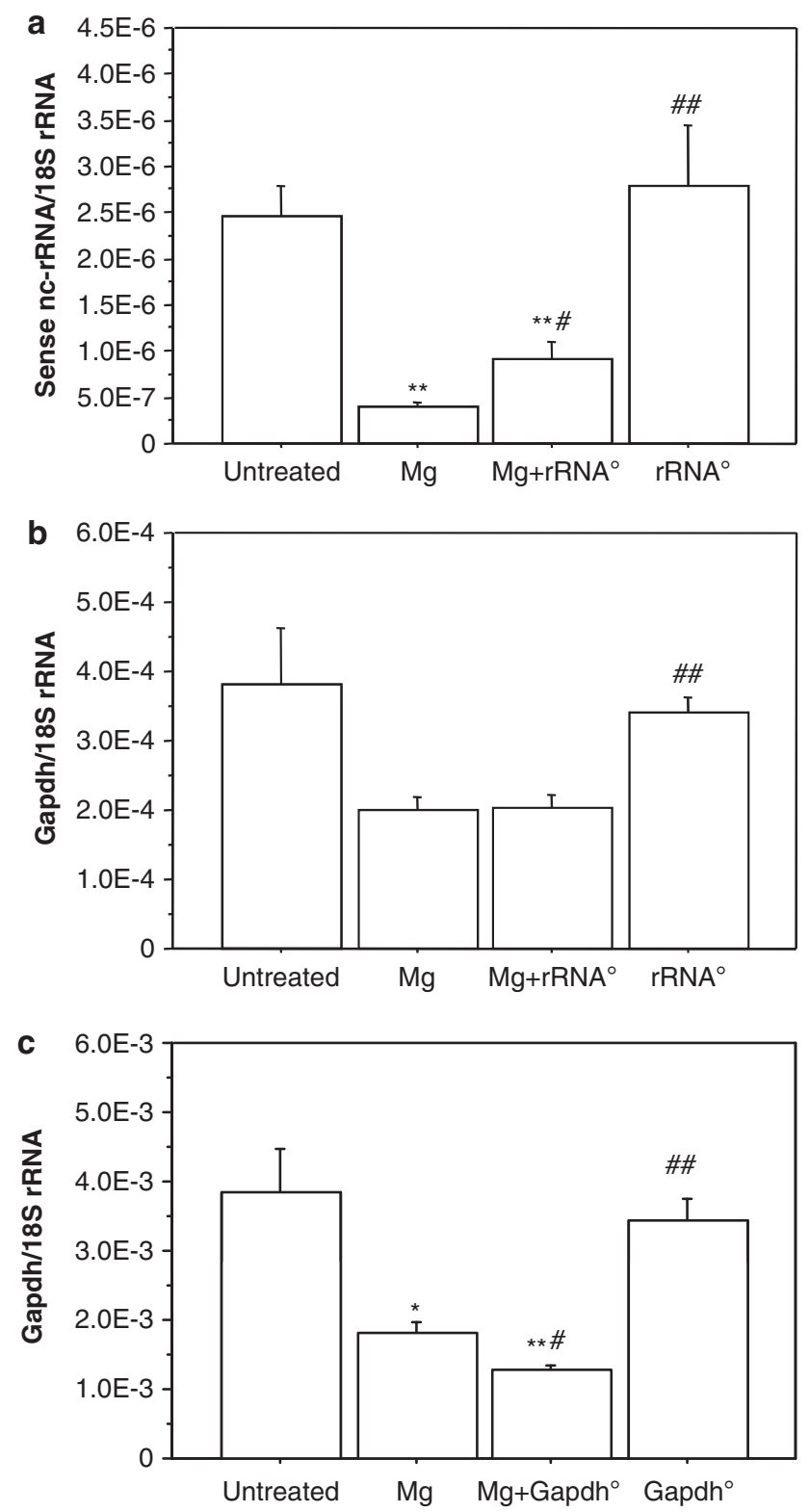

d

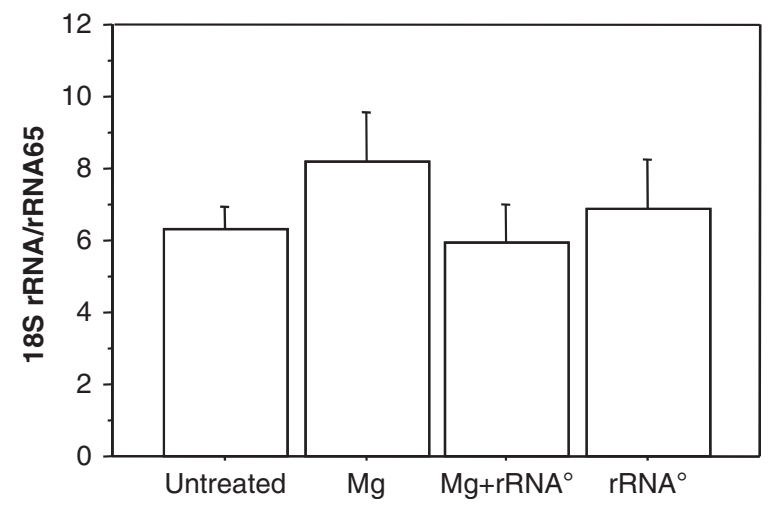

regions, +273 to +556 and around +3999 nucleotides. It is noteworthy that these cleavage-ligation sites also correspond to the RT-PCR region (nucleotides -241 to +110 ) showing sense nc-rRNA instability or catalysis in the previous section.

Mutual upregulation of bidirectional nc-rRNAs and induction of cell death. Locked nucleic acid (LNA)-DNA gapmer oligonucleotides (Supplementary Figure S1a) complementary to four regions around the putative catalytic sites (Table 1) were introduced to mouse lung cells to test if endogenous nc-rRNAs can be stabilized as seen in the in vitro assay. Initial examination of adherent cells $24 \mathrm{~h}$ after LNA transfection did not detect upregulation of target nc-r RNAs specific to any complementary LNA (Supplementary Figure S1b). However, we noticed that LNA1-S targeting the $-103 /-85$ region of antisense nc-rRNAs apparently induced more cell detachment compared with vehicle and LNA1-AS, a complementary strand of the LNA1-S (Figure 4a). Only about $50 \%$ viable $\mathrm{A} 5$ and $\mathrm{E} 9$ cells remained after transfection with LNA1-S, whereas $>80 \%$ viability was seen for the LNA1-AS, relative to vehicle treatment (Figure $4 \mathrm{~b}$ ). This prompted us to collect detached cells from these two treatments for RNA analysis. The cell detachment at $24 \mathrm{~h}$ in LNA1-AS and vehicle treatments were rare and insufficient total RNA precluded subsequent assay for nc-rRNAs despite the effort to pool detached cells from multiple plates. For this reason, adherent and detached cells were collected at $48 \mathrm{~h}$ after two LNA transfections with $24 \mathrm{~h}$ apart. Strikingly, over 15-fold mutual upregulations of sense and antisense ncrRNAs were consistently detected in massively detached A5 and E9 cells induced by the LNA1-S (Figure 4c). Similar changes were also observed in detached cells from LNA1AS, vehicle, and untreated samples, however, few cells were detached and significantly higher levels of nc-rRNAs in LNA1-AS sample relative to LNA1-S did not translate to more cell death. This implies that the LNA1-AS-induced cell detachment may represent nonspecific or spontaneous cell death as seen in vehicle and untreated samples. Taken together, these observations strengthen the proposals that sense and antisense nc-rRNAs follow a feed-forward mechanism to regulate each other via suppression of RNA catalysis and their accumulations are involved in cell destruction. These assumptions are further supported by small changes of nc-rRNA levels in adherent cells (Figure 4c). Slight but significant increases of the nc-rRNAs in adherent cells from LNA1-S treatment compared with either vehicle or LNA1-AS may have been a result of upregulated transcripts from dying cells that have not yet detached.

Figure 3 Ribozyme-like characteristics of sense nc-rRNAs. Relative level of sense nc-rRNAs to the $18 S$ rRNA reference (a), of the Gapdh to the $18 S$ rRNA (b), and of the 18S rRNA to a 65-bp rRNA (d) in the presence/absence of $r R N A$-specific complementary oligonucleotides (rRNA ${ }^{\circ}$ ). (c) Relative level of the Gapdh to the $18 \mathrm{~S}$ rRNA in the presence/absence of Gapdh-specific complementary oligonucleotides $\left(\right.$ Gapdh $\left.^{\circ}\right) .{ }^{*} P<0.05,{ }^{* *} P<0.01$, compared with untreated sample after treatment of $\mathrm{MgCl}_{2}, \mathrm{MgCl}_{2}+$ oligonucleotides, or oligonucleotides alone. ${ }^{\#} P<0.05,{ }^{\#} P<0.01$, compared with $\mathrm{MgCl}_{2}$-treated samples. Error bars denote \pm S.E. 
Table 1 Cleavage-ligation products of sense nc-rRNA transcripts in mouse lung epithelial cells

\begin{tabular}{|c|c|}
\hline Freq. $^{a}$ & Sequence $^{\mathrm{b}}$ \\
\hline 2 & $\begin{array}{l}\text { AGGCCGAGGGAAAGCTATGGGCGCGGTTTTCTTTTCTCTTCCCGGTCTTTCTTCCACATGCC } \\
\text { TCCCGAGTGCATTTCTTTTTTGTTTTTTTTCTTTTTTTTTGGGGGGGGAGGTGGAGAGTCCC } \\
\text { GAGTACTTCACTCCTGTCTGCGGTGTCCAAGTGTTCATGCCACGTGCCTCCCGAGTGCACTT } \\
\text { TTTTTTTGTGGCAGTCGCTCGTTGTGTTCTCTTGTTCTGTGTCTGCCCGTATCAGTAAC }\end{array}$ \\
\hline 1 & $\begin{array}{l}\text { AGGCCGAGGGAAAGCTATGGGCGCGGTTTTTCTTTCATTGACCTGTCGCTCTTTTCTCTTCC } \\
\text { CGGTCTTTCTTCCACATGCCTCCCGAGTGCATTTCTTTTTGTTTTTTTTCTTTTTTTTTGGG } \\
\text { GGGGGAGGTGGAGAGTCCCGAGTACTTCACTCCTGTCTGCGGTGTCCAAGTGTTCATGCCAC } \\
\text { GTGCCTCCCGAGTGCACTTTTTTTTTGTGGCAGTCGCTCGTTGTGTTCTCTTGTTCTGTGTC } \\
\text { TGCCCGTATCAGTAAC }\end{array}$ \\
\hline
\end{tabular}

1 AGGCCGAGGGAAAGCTATGGGCGCGGTTTTCTTTCATTGACCTGTCGGTCTTATCAGTTCTC CCGGTTGGAATGGTGGAGCCAGCTGTGGTTGAGGGCCGCCTTATTTCGGCTCAC . . .$$
5^{\prime} \text {-join }{ }^{\mathrm{c}}
$$$$
-213
$$$$
3^{\prime} \text {-join }{ }^{d}
$$$$
\text { Gape }
$$

$+366$

1 AGGCCGAGGGAAAGCTATGGGCGCGGTTTTCTTTCATTGACCTGTCGGTCTTATCAGTTCTC CCGGTTGTCAGGTCGACCAGTTGTTCCTTTGAGGTCCGGTTCTTTTCGTTATGGGGTCATTT TTGGGCCACCTCCCCAGGTATGACTTCCAGGTATTCTTTGTGGCAGTCGCTCGTTGTGTTCT CTTGTTCTGTGTCTGCCCGTATCAGTAAC

11 AGGCCGAGGGAAAGCTATGGGCGCGGTTTTCTTTCATTGACCTGTCGGTCTTATCAGTTCTC CCGGTTGTGGCAGTCGCTCGTTGTGTTCTCTTGTTCTGTGTCTGCCCGTATCAGTAAC

2 AGGCCGAGGGAAAGCTATGGGCGCGGTTTTCT GGCCCAGGTGTCTGCCCGTATCAGTAAC

3 AGGCCGAGGGAAAGCTATGGGCGCGGTTTTCTTTCATTGACCTGTCGGTCTTATCAGTTCTC TTGTTCTGTGTCTGCCCGTATCAGTAAC

1 AGGCCGAGGGAAAGCTATGGGCGCGGTTTTCTTTCATTGACCTGTCGGTCTTATCAGTTCTC CTGTTCTGTGTCTGCCCGTATCAGTAAC

4 AGGCCGAGGGAAAGCTATGGGCGCGGTTTTCTTTCATTGACCTGTCGGTCTTATCAGTTCTC CTGTCTGCGGTGTCCAAGTGTTCATGCCACGTGCCTCCCGAGTGCACTTTTTTTTGTGGCAG TCGCTCGTTGTGTTCTCTTGTTCTGTGTCTGCCCGTATCAGTAAC

5 AGGCCGAGGGAAAGCTATGGGCGCGGTTTTCTTTCATTGACCTGTCGGTCTTATCAGTTCTC CCGGTTGTCAAGTCGACCAGTTGTTCCTTTGAGGTCCGGTTCTCTTGTTCTGTGTCTGCCCG TATCAGTAAC

1 AGGCCGAGGGAAAGCTATGGGCGCGGTTTTCTTTCATTGACCTGTCGGTCTTATCAGTTCTC CCGGTTGTGTTCTCTTGTTCTGTGTCTGCCCGTATC

1 AGGCCGAGGGAAAGCTATGGGCGCGGTTTTCTTTCATTGACCTGTCGGTCTTATCAGTTCTC CC CGTTGTGTTCTCTTGTTCTGTGTCTGCCCGTATCAGTAAC

1 AGGCCGAGGGAAAGCTATGGGCGCGGTTCTCTTGTTCTGTGTCTGCCCGTATCAGTAAC

1 AgGCCGAGGGAAAGCTATGGGCGCGGTTTTCTTGTTCTGTGTCTGCCCGTATCAGTAAC

2 GAGGGAAAGCTATGGGCGCGGTTTTCTTTCATTGACCTGTCGCTCGTTGTGTTCTCTTGTTC TGTGTCTGCCCGTATCAGTAAC

1 AGGCCGAGGGAAAGCTATGGGCGCGGTTTTCTTTCATTGACCTGTCGGTCTTATCAGTTCTC CGGGTTGTCAGGTCGACCAGTTGTTCCTTTGAGGTCCGGTTCTTTTCGTTATGGGGTCATTT TTGGGCCACCTCCCCAGGTATGACTTCCAGGTATTCTCTGTGGCCTGTCACTTTCCTCCCTG TCTCTTTTATGCTTGTGTTCTCTTGTTCTGTGTCTGCCCGTATCAGTAAC

3 AGGCCGAGGGAAAGCTACCTGGTTGATCCTGCCAGTAGCATATGCTTGTCTCAAAGATTAAG CCATGCATGTCTAAGTÄCGCACGGC

$\begin{array}{lll}-176 & +527 & 699 \\ -210 & +556 & 766 \\ -186 & +549 & 729 \\ -179 & +550 & 729 \\ -183 & +471 & 650 \\ -142 & +548 & 685 \\ -177 & +543 & 715 \\ -178 & +537 & 715 \\ -217 & +546 & 760 \\ -209 & +551 & 760 \\ -199 & +535 & 730 \\ -44 & +544 & 583\end{array}$

\footnotetext{
${ }^{a}$ The frequency of occurrence. ${ }^{b}$ Solid lines: primer sequences; dotted lines: cleavage-ligation regions but difficulty in assigning to a specific nucleotide; boxed sequence: does not match to either $5^{\prime}$ or $3^{\prime}$ sequences. ${ }^{\mathrm{C}}$ The nucleotide immediately adjacent to the $5^{\prime}$-end of sequences with dotted lines. ${ }^{\mathrm{d}}$ The nucleotide

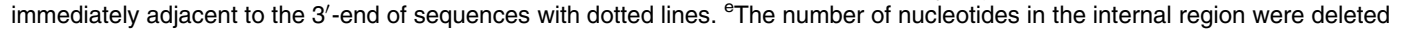




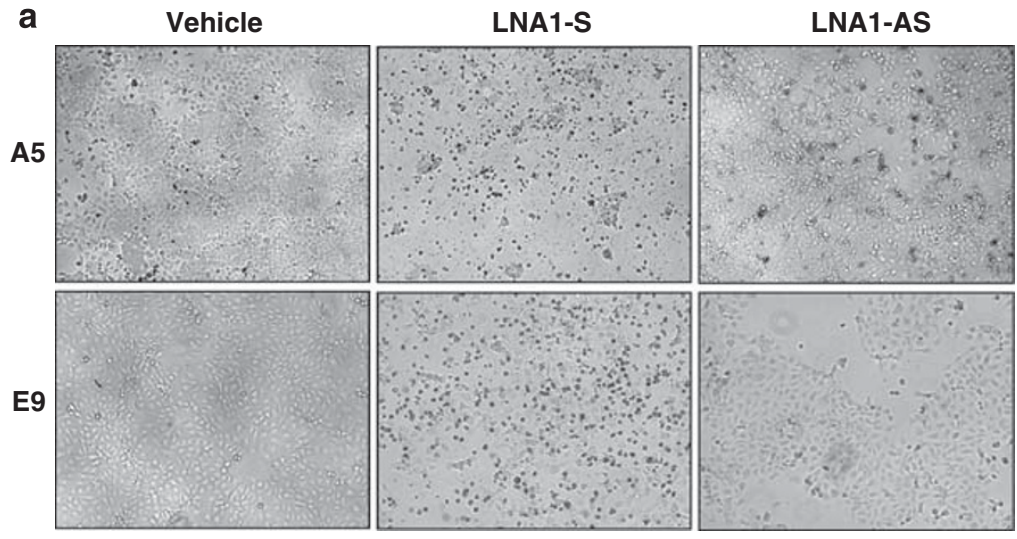

b

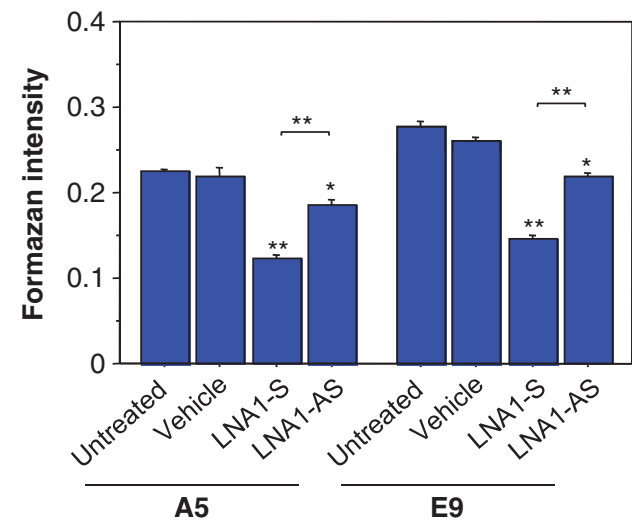

C Adherent
\begin{tabular}{|c|c|c|c|c|c|c|c|}
\hline Transcript & Cell & LNA1-S & LNA1-AS & LNA1-S & LNA1-AS & Vehicle & Untreated \\
\hline Sense & A5 & $+2.5^{*}, \mathrm{P}<0.0001$ & $+1.4, \mathrm{P}=0.0043$ & $+28.5^{*}, \mathrm{P}<0.0001$ & $+345.2, \mathrm{P}=0.0157$ & $+6.8, \mathrm{P}=0.0019$ & $+5.4, \mathrm{P}=0.0028$ \\
nc-rRNA & $\mathrm{E} 9$ & $+1.6^{*}, \mathrm{P}=0.0015$ & $-1.3, \mathrm{P}=0.0066$ & $+27.8, \mathrm{P}<0.0001$ & $+22.6, \mathrm{P}=0.0020$ & $+7.3, \mathrm{P}=0.0014$ & $+8.2, \mathrm{P}=0.0009$ \\
\hline Antisense & $\mathrm{A} 5$ & $+1.4^{*}, \mathrm{P}=0.0062$ & $+1.4, \mathrm{P}=0.0184$ & $+16.8^{*}, \mathrm{P}<0.0001$ & $+679.8, \mathrm{P}=0.0116$ & $+9.2, \mathrm{P}=0.0015$ & $+8.8, \mathrm{P}=0.0003$ \\
\cline { 2 - 9 } nc-rRNA & $\mathrm{E} 9$ & $+1.1^{*}, \mathrm{P}=0.6023$ & $-1.3, \mathrm{P}=0.0303$ & $+21.3^{*}, \mathrm{P}<0.0001$ & $+47.6, \mathrm{P}<0.0001$ & $+7.1, \mathrm{P}<0.0001$ & Undetectable \\
\hline Leader & $\mathrm{A} 5$ & $+2.1^{*}, \mathrm{P}<0.0001$ & $+1.3, \mathrm{P}=0.0043$ & $+5.0^{*}, \mathrm{P}<0.0001$ & $-1.0, \mathrm{P}=0.8161$ & $-1.5, \mathrm{P}=0.0013$ & $-1.2, \mathrm{P}=0.0397$ \\
rRNA & $\mathrm{E} 9$ & $+1.9^{*}, \mathrm{P}=0.0024$ & $-1.3, \mathrm{P}=0.0023$ & $+3.8, \mathrm{P}=0.0106$ & $-2.2, \mathrm{P}=0.0007$ & $-3.7, \mathrm{P}=0.0038$ & $-3.8, \mathrm{P}=0.0034$ \\
\hline 5'ETS & $\mathrm{A} 5$ & $+1.4^{*}, \mathrm{P}<0.0001$ & $+1.2, \mathrm{P}=00870$ & $+17.0, \mathrm{P}=0.0001$ & $+1.1, \mathrm{P}=07008$ & $+2.6, \mathrm{P}<00001$ & $+3.1, \mathrm{P}<0.0001$ \\
\cline { 2 - 9 } rRNA & $\mathrm{E} 9$ & $+1.3^{*}, \mathrm{P}=0.0075$ & $-1.2, \mathrm{P}=0.0004$ & $+25.2^{*}, \mathrm{P}<0.0001$ & $+6.6, \mathrm{P}<0.0001$ & $+4.5, \mathrm{P}=0.0051$ & $+3.2, \mathrm{P}=0.0003$ \\
\hline
\end{tabular}

Figure 4 The levels of nc-rRNAs, pre-rRNAs, and cell viability after LNA1-S and LNA1-AS treatments. (a) Abundant dead cells, represented by the dense granules under microscope, were immediately recognizable in cells $24 \mathrm{~h}$ after transfection with LNA1-S ( $\times 50$ magnification). (b) The viable cells relative to vehicle treatment were also significantly reduced at $24 \mathrm{~h}$ after LNA treatments and the effect was more pronounced for LNA1-S, compared with LNA1-AS $\left({ }^{*} P<0.05 ;{ }^{* *} P<0.0005\right)$. Error bars denote \pm S.E. (c) Heat map for the nc-rRNA and pre-rRNA profiles in A5 and E9 cells at $48 \mathrm{~h}$ following two transfections of LNA gapmer with $24 \mathrm{~h}$ apart. Statistically significant changes in reference to corresponding transcripts in vehicle-treated adherent cells were colored in red for upregulation and in green for downregulation. Numbers indicate foldchanges and $P$-values. ${ }^{*} P<0.05$ for transcript differences between LNA1-S and LNA1-AS treatment in adherent and detached cells

Because nc-rRNAs are overlapping and/or complementary to primary $r R N A$, their mutual upregulations may potentially alter $r R N A$ biogenesis. Indeed, all detached cells were frequently detected with accumulation of pre-rRNAs, revealed by the leader- or $5^{\prime}$ ETS-containing transcripts corresponding to the $47 S$ and/or $45 S$ rRNAs during $r R N A$ biogenesis (Figure 4c). The detached cells from LNA1-S treatment elevated both $47 S$ and $45 S$ rRNAs, whereas others showed accumulation at the $45 S$ rRNA step. The LNA1-S appears to perturb $r R N A$ biogenesis via a mechanism distinct from LNA1AS and spontaneous cell detachment.

Cell death via apoptotic and nonapoptotic mechanisms. It is known that disruption of $r R N A$ biogenesis triggers apoptosis. $^{20}$ To test this hypothesis in LNA-treated cells, internucleosomal DNA breaks, indicative of apoptosis, as well as cell cycle were examined. The LNA1-S indeed enhanced apoptosis in $\mathrm{A} 5$ at $24 \mathrm{~h}$, revealed by a steam-like appearance of cell population with intense labeling of internucleosomal DNA breaks in the scatter plot (Figure 5a, upper left and upper right fractions). Growth in A5 was also suppressed, represented by reduction in the S-phase fraction of cell cycle (Figure 5b). The apoptotic cell death and growth suppression in A5 were clearly more prominent in the LNA1-S treatment than the LNA1-AS, excluding possible effects simply due to the presence of oligonucleotides and delineating the mechanism of cell inhibition upon stabilization of antisense nc-rRNAs. In contrast, the apoptotic response in $\mathrm{E} 9$ at $24 \mathrm{~h}$ was insignificant (Figure 5a) and much lower than the estimated $50 \%$ reduction of cell viability (Figure $4 \mathrm{~b}$ ), providing evidence that LNA1-S also mediates death via mechanisms other than apoptosis. Neither LNA1-S nor LNA1-AS exerted any effect on cell cycle in E9 (Figure 5b).

To further demonstrate that antisense nc-rRNAs are potential targets for induction of apoptosis, additional complementary LNAs listed in Supplementary Figure S1a were tested. As shown in Figure 6a, LNA4-S, complementary to the $+484 /+502$ region of antisense nc-rRNAs, was more potent than other LNAs targeting sense nc-rRNAs to induce the steam-like appearance of apoptotic cell population in the scatter plot of A5 cells. The apoptotic responses of E9 cells to these LNAs including LNA4-S were small at $24 \mathrm{~h}$ (Figure 6a), similar to the LNA1-S treatment (Figure 5a). The 48-h treatment of LNA1-S and LNA4-S was required to trigger extensive apoptosis in E9 (Figure 6b). The LNA4-S remained highly apoptogenic in A5 at $48 \mathrm{~h}$. In contrast, the 48-h incubation with LNA1-S reduced apoptotic fractions in A5 compared with 24-h treatment in Figure $5 \mathrm{a}$, suggesting that cell death switches from apoptotic to nonapoptotic mechanism after prolonged LNA1-S treatment. Similar striking 
a
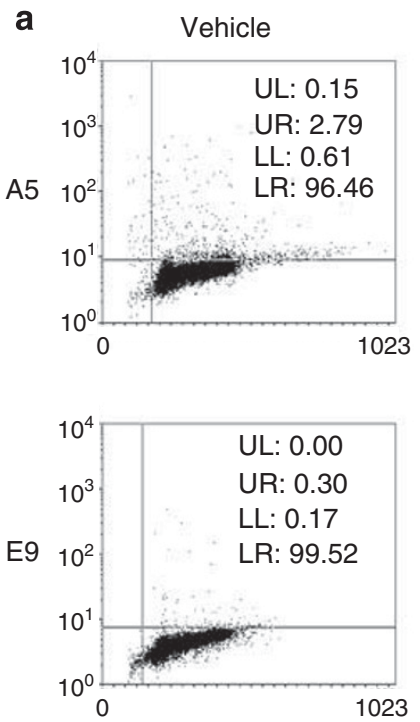

b

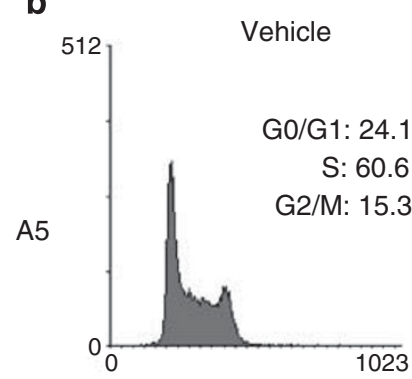

LNA1-S
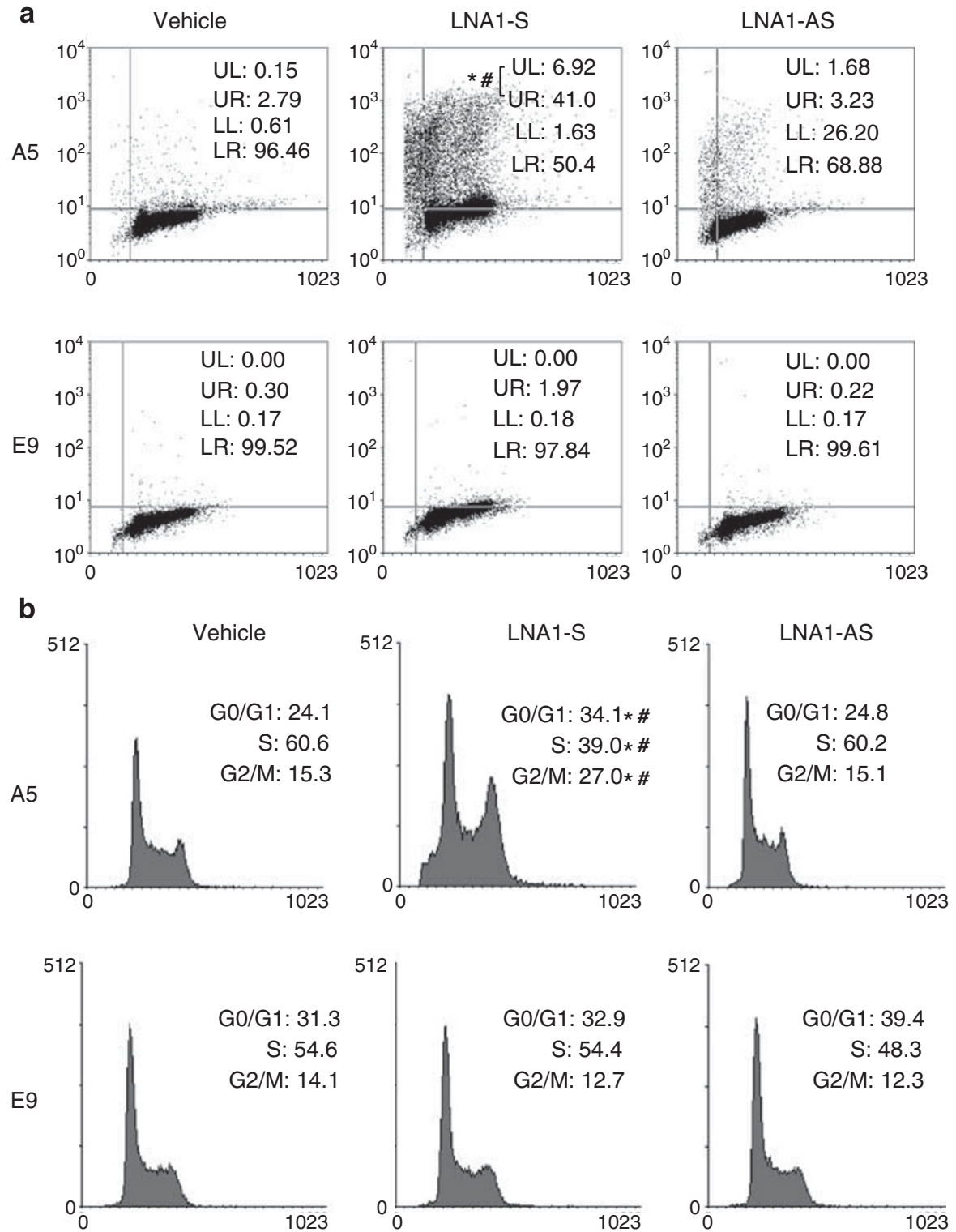

Figure 5 Effects on apoptosis and cell cycle at $24 \mathrm{~h}$ after single transfection of LNAs. (a) The upper left (UL) and upper right (UR) fractions represent estimated apoptotic cell populations. The lower left (LL) fraction contains nonapoptotic/fragmented cells and the lower right (LR) region corresponds to unaffected cells (X-axis: propidium iodide intensity; Y-axis: fluorescein intensity in log scale). (b) Histogram of cell cycle shows G0/G1, S, and G2/M phases (X-axis: propidium iodide intensity; Y-axis: cell count). The large sub-G0/G1 population in LNA1-S-treated A5 cells is consistent with the marked increase of apoptotic cells in (a). ${ }^{\#} P<0.05$, compared with vehicle; ${ }^{*} P<0.05$, compared with LNA1-AS

cell-killing efficacy between LNA4-S and LNA1-S prompted us to examine whether LNA4-S induces stabilizations of bidirectional nc-rRNAs as seen for the LNA1-S. Detached cells from the LNA4-S treatment in fact carried extremely high levels of sense and antisense nc-rRNAs, as well as pre-rRNAs, whereas only small changes were observed in adherent cells (Figure $6 \mathrm{c}$ ), confirming the same feed-forward mechanism utilized by LNA4-S and LNA1-S.

Concomitant upregulation of bidirectional nc-rRNAs and cell death after LNA1-S and LNA4-S treatments raised the possibility that nc-rRNAs may function as death mediators. To test this hypothesis, in-vitro synthesized nc-rRNAs in sense and antisense orientations (Figure 7) were transfected into A5 and E9 cells. Initial trials of $0.5-4 \mathrm{nM} \mathrm{nc-rRNAs} \mathrm{did} \mathrm{not}$ appear to be cytotoxic in both cell lines $48 \mathrm{~h}$ after transfection. Differential apoptotic and nonapoptotic death responses were detected following nearly 48-h treatments with $8 \mathrm{nM} 1 \mathrm{~S}, 2 \mathrm{~S}$, $1 \mathrm{AS}$, and/or 2AS nc-rRNAs (Figure 7). These nc-rRNAs significantly increased apoptotic and nonapoptotic cell killing compared with NS nc-rRNA, which was not originally present in mouse lung cells (Figure 1), strengthening the role of endogenous nc-rRNAs in regulation of the multiple death pathways.

\section{Discussion}

Screening of noncoding transcripts within the rDNA loci has identified extensive bidirectional cis-nc-rRNAs. The extent of 
a
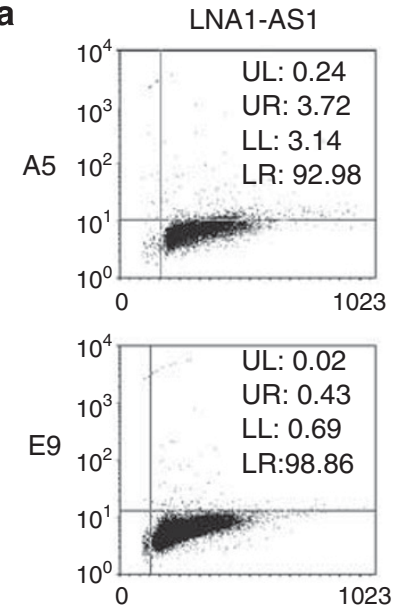

b
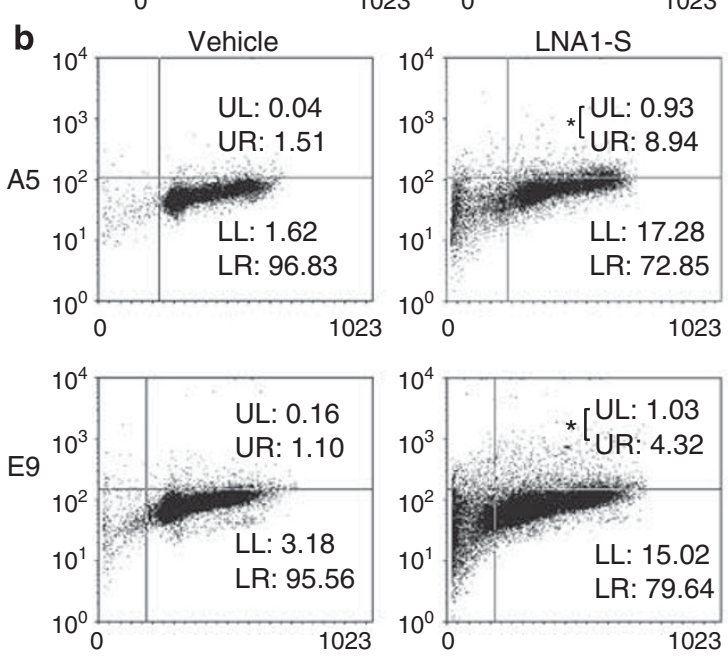

LNA2-AS
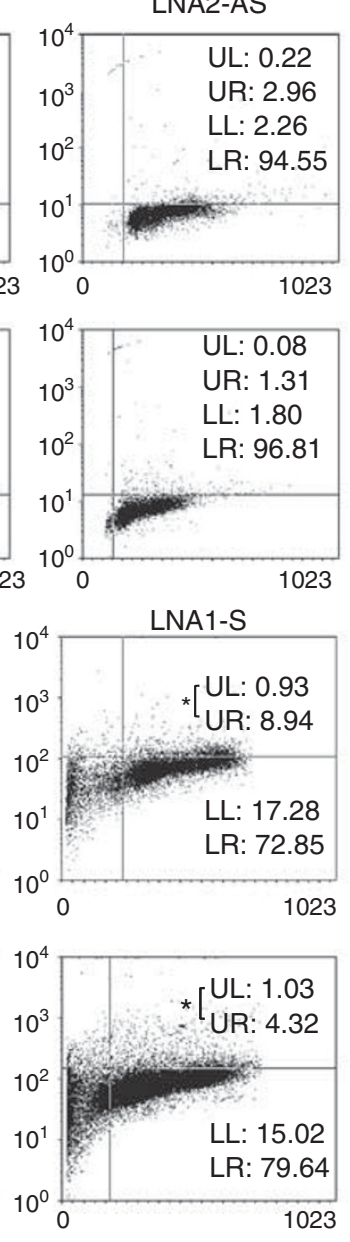

.
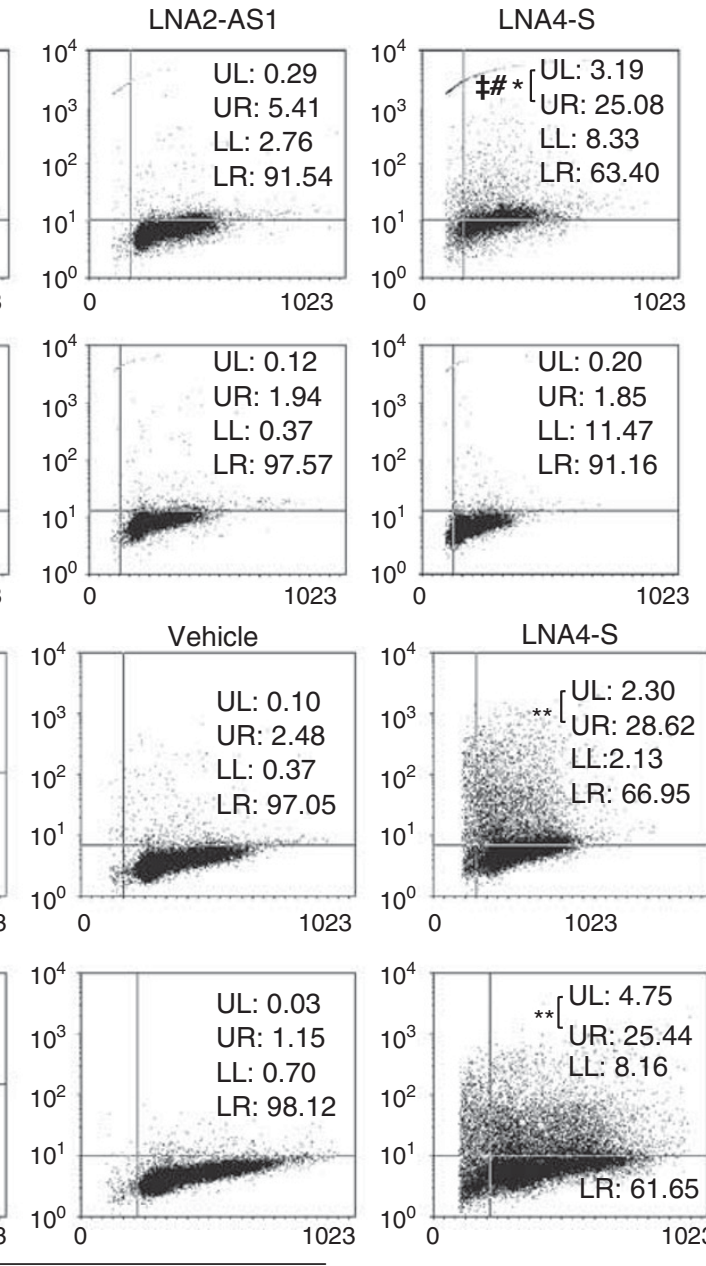
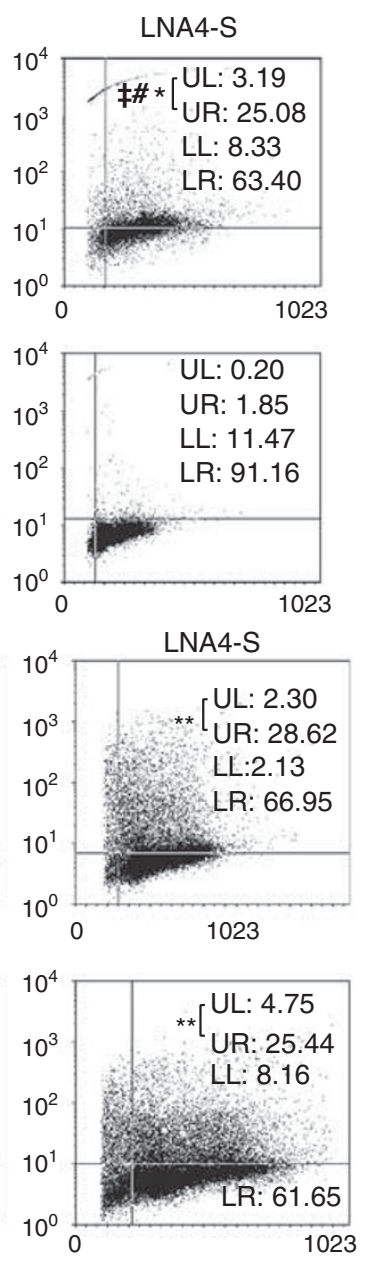

\begin{tabular}{|c|c|c|c|}
\hline Transcript & Cell & Adherent & Detached \\
\hline \multirow{2}{*}{$\begin{array}{c}\text { Sense } \\
\text { nc-rRNA }\end{array}$} & A5 & $+2.0, P<0.0001$ & $+53.4, P<0.0001$ \\
\hline & E9 & $+1.1, P=0.1528$ & $+77.9, \mathrm{P}<0.0001$ \\
\hline \multirow{2}{*}{$\begin{array}{l}\text { Antisense } \\
\text { nc-rRNA }\end{array}$} & A5 & $-1.1, P=0.5510$ & $+97.6, P=0.0002$ \\
\hline & E9 & $-1.6, P<0.0001$ & $+42.5, P<0.0001$ \\
\hline \multirow{2}{*}{$\begin{array}{c}\text { Leader } \\
\text { rRNA }\end{array}$} & A5 & $+1.3, P=0.1486$ & $+5.3, P=0.0002$ \\
\hline & E9 & $+1.4, P<0.0001$ & $+2.8, P<0.0001$ \\
\hline \multirow{2}{*}{$\begin{array}{l}5 \text { 'ETS } \\
\text { rRNA }\end{array}$} & A5 & $+1.5, P=0.0024$ & $+65.3, P<0.0001$ \\
\hline & E9 & $+1.4, P=0.0004$ & $+64.6, P<0.0001$ \\
\hline
\end{tabular}

Figure 6 The apoptotic responses of cells to additional LNAs and treatment schedule. (a) Effects at $24 \mathrm{~h}$ following single transfection of LNAs. The UL and UR fractions represent estimated apoptotic cell populations. The LL fraction contains nonapoptotic/fragmented cells and the LR region corresponds to unaffected cells (X-axis: propidium iodide intensity; Y-axis: fluorescein intensity in log scale). The steam-like appearance in the UL and UR fractions of LNA4-S-treated A5 cells indicates large apoptotic population. ${ }^{\ddagger} P<0.05,{ }^{\#} P<0.05$, and ${ }^{*} P<0.05$, compared with LNA1-AS1, LNA2-AS, and LNA2-AS1, respectively. (b) The responses at $48 \mathrm{~h}$ following two transfections of LNAs with $24 \mathrm{~h}$ apart. ${ }^{*} P<0.05$ and ${ }^{* *} P<0.01$, compared with vehicle control. (c) Heat map for the nc-rRNA and pre-rRNA profiles in A5 and E9 cells at $48 \mathrm{~h}$ following two transfections of LNA4-S gapmer. Statistically significant changes in reference to corresponding transcripts in vehicle-treated adherent cells were colored in red for upregulation and in green for downregulation. Numbers indicate fold-changes and $P$-values

antisense nc-rRNAs is consistent with a recent report. ${ }^{21}$ This $^{2}$ raises a possibility that the $r R N A$ biogenesis, a key process to sustain cell growth and survival, ${ }^{17,18}$ is regulated by nc-rRNAs at multiple sites. Both sense and antisense nc-rRNAs are discontinuous, suggesting that transcription of these multiple nc-rRNAs may be initiated independently, though posttranscriptional RNA processing cannot be excluded. Sense
nc-rRNAs, transcribed from regions upstream to the $r R N A$ transcription start site, have been described before ${ }^{12,13}$ and thought to be initiated from a spacer promoter at -2026 to -1996 region in mice (GenBank BK000964). Several termination sites have been also reported, including -170 and -182 nucleotides. ${ }^{12,13}$ In this study, we detected a sense transcript covering the -2547 region and two long sense 

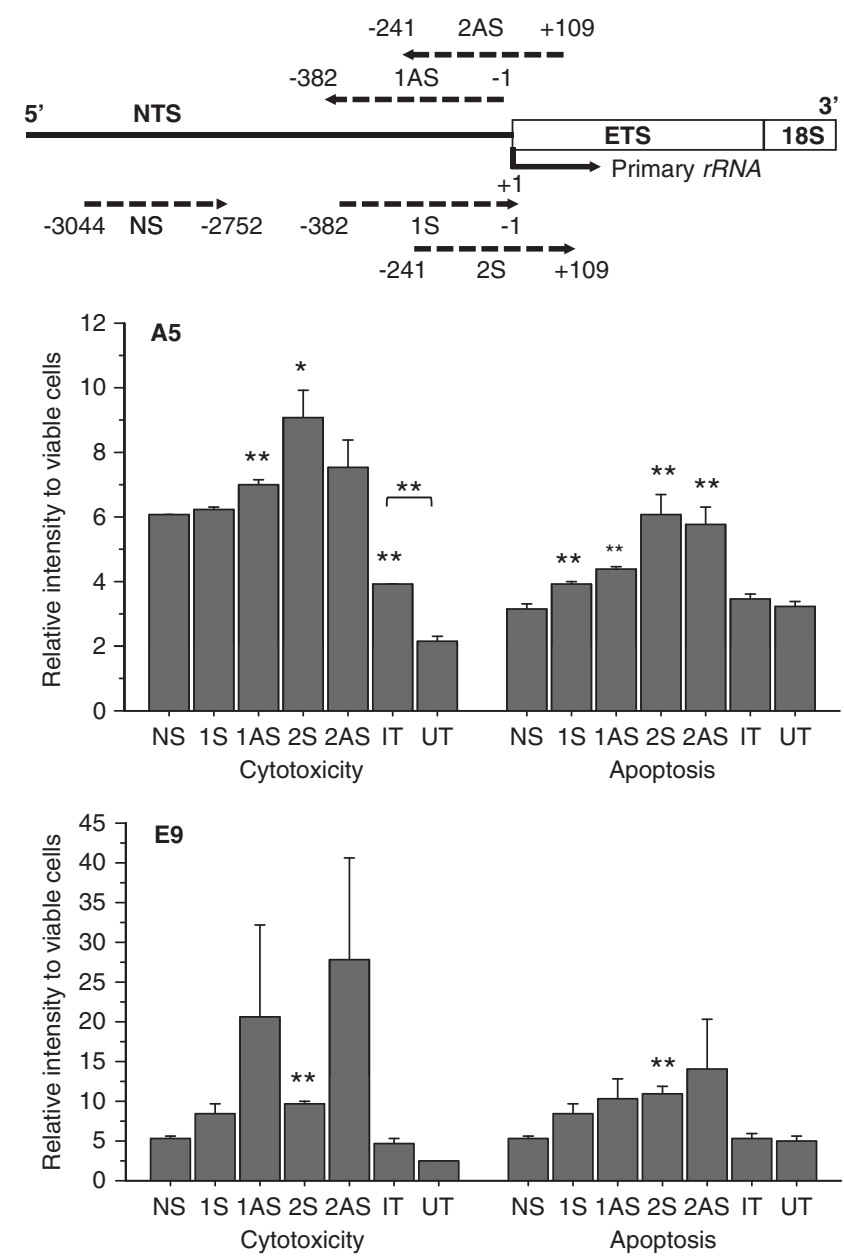

Figure 7 Direct induction of cytotoxicity and apoptosis by in vitro-synthesized nc-rRNAs. Cytotoxicity and apoptosis relative to viability was illustrated for cells (A5 and E9) $45 \mathrm{~h}$ after single transfection of selected nc-rRNA transcripts (sense: $1 S$ and 2S; antisense: $1 A S$ and 2AS, see upper panel for their locations). NS, nonspecific nc-rRNA control; IT, TransIT transfection vehicle control; UT, untreated. ${ }^{*} P<0.05,{ }^{* *} P<0.01$, compared with the NS control. Error bars denote \pm S.E. from triplicate samples. Error bars denote \pm S.E.

nc-rRNAs encompassing regions of -321 to +7295 and -238 to +7246 . Transcription of sense nc-rRNA across the primary $r R N A$ start site is conserved in humans, ${ }^{11}$ supporting the idea that nc-rRNAs are biologically important in mammalian cells. Also, application of multiple capture probes and complementary oligonucleotides across gene locus is essential to prevent catalysis of nc-rRNAs.

Self-catalytic $\mathrm{R} 2$ retrotransposons in the $28 S \mathrm{r} R \mathrm{NA}$ region have been described recently. ${ }^{22}$ The $r R N A$ component of the ribosome is known to catalyze translation via its peptidyl transferase activity. ${ }^{23,24}$ Observation of the ribozyme-like properties in nc-rRNAs is very intriguing and supports that $r R N A s$ and nc-rRNAs are highly reactive with diverse biological functions. Also, these nc-rRNAs and perhaps noncoding RNAs in other genes may require self-catalytic RNA processing before carrying out their putative functions. Suppression of ribozyme activity may consequently lead to unfavored phenotype, such as cell death.
As predicted, introduction of complementary LNAs stabilizes sense and antisense nc-rRNAs in mouse lung cells and induces cell death. Mutual upregulation of the bidirectional nc-rRNAs is consistent with inhibition of ribozyme-like activity by complementary sequences, LNA1-S/antisense ncrRNAs, LNA4-S/antisense nc-rRNAs, and antisense/sense nc-rRNAs pairs. The ribozyme-like properties of the sense nc-rRNAs have been demonstrated in the in-vitro assay. Stabilization of antisense nc-rRNAs after LNA1-S and LNA4-S treatments provides the evidence that antisense transcripts also carry ribozyme-like properties. The bidirectional nc-rRNAs apparently act through a feed-forward mechanism upon LNA treatment to regulate each other and to induce cell death. Viable cells retain basal levels of nc-rRNAs. Similar feed-forward mechanisms between sense and antisense transcripts have been described before, ${ }^{25,26}$ but it is not clear whether any ribozyme activity is involved in those genes.

Several sense and antisense nc-rRNAs are known to regulate $r R N A$ biogenesis. Ectopic expression of an intergenic sense nc-rRNA from -142 to -40 nucleotides has been shown to displace nucleolar remodeling complex and to upregulate the $47 S$ rRNA in mouse fibroblast cells. ${ }^{14}$ Suppression of $r R N A$ transcription but accumulation of the 45S rRNA by sense and antisense nc-rRNAs from the $5^{\prime}$ ETS region have been reported in human cancer cells. ${ }^{27}$ In the current study, we demonstrate that upregulation of endogenous nc-rRNAs can be achieved by introduction of antisense oligonucleotides, a useful tool for future in vivo experiments. Strikingly, stabilization of endogenous nc-rRNAs induces cell death as well as upregulations of $47 S$ and/or $45 S$ rRNAs. The role of nc-rRNA stabilization in cell death is further supported by concurrent accumulations of pre-rRNA levels and nc-rRNAs in spontaneously detached cells from vehicle and untreated controls (Figure 4c). LNA1-S and LNA4-S are not complementary to pre-rRNAs, and therefore, the perturbation of $r R N A$ biogenesis is likely mediated by direct interaction with antisense nc-rRNAs. The two distinct prerRNA profiles in LNA1-S and LNA4-S treatments versus LNA1-AS and controls suggest that different sets of antisense nc-rRNAs may be involved in regulation of $r R N A$ biogenesis. Taken together, these observations strengthen the proposal that antisense nc-rRNAs, upregulated by a feed-forward mechanism involving ribozyme inhibition, serve as death mediators by exerting interferences at different steps of $r R N A$ biogenesis.

Activation of the cell-death pathway via stabilizations of both sense and antisense nc-rRNAs may represent a novel anticancer strategy. The death mechanism varies depending on cell type and treatment schedule. Apoptotic response is readily detectable in A5 with a single LNA dosing, whereas E9 requires multiple treatments. Mechanisms other than apoptosis dominate the LNA-induced cell death in E9, supporting that nc-rRNAs mediate cell destruction through multiple pathways. This is further confirmed by detection of apoptotic and nonapoptotic death in A5 and E9 cells following transfection of synthetic nc-rRNAs (Figure 7), consistent with a recent report using human cells. ${ }^{28}$ The preferential killing of LNAs complementary to antisense nc-rRNAs, compared with those pairing with sense nc-rRNAs, may be explained 
by better accessibility of antisense nc-rRNAs than their sense counterparts to complementary oligonucleotides, however, this remains to be tested. Many anticancer drugs have been shown to promote cell death by disrupting different stages of the $r R N A$ biogenesis in various cancer types. ${ }^{29-31}$ Among them, $5^{\prime}$-fluorouracil is known to carry a property to inhibit ribozymatic self-cleavage in mammalian cells. ${ }^{32}$ Identification of ribozyme-like properties in nc-rRNAs represents a new opportunity to screen small molecules for cancer-selected treatment. In conclusion, the current findings of ribozymelike nc-rRNAs and their functions to mediate cell death, via apoptotic and non-apoptotic pathways, and/or growth suppression provide proof of principle that nc-rRNAs are promising targets for the discovery of potent anticancer drugs.

\begin{abstract}
Materials and Methods
Cell lines. Spontaneously transformed E9 and A5 lung-cancer cell lines of BALB/c mouse origin were obtained from Dr. A Malkinson, the University of Colorado, and Dr. S Jakowlew, the National Cancer Institute, respectively. E9 cells were cultured in CMRL 1066 medium and A5 cells were maintained in Dulbecco's modified Eagle's medium (Invitrogen, Carlsbad, CA, USA), supplemented with $4 \mathrm{mM}$ glutamine, $100 \mu \mathrm{g} / \mathrm{ml}$ penicillin/streptomycin, and $10 \%$ fetal calf serum. Cells in T-150 flasks were grown in a $37^{\circ} \mathrm{C}$ incubator (Thermo Scientific, Waltham, MA, USA) provided with $7 \%$ carbon dioxide air. Near-confluent cells were harvested with $0.05 \%$ trypsin-EDTA and then washed with phosphate-buffered saline before storage at $-70^{\circ} \mathrm{C}$.
\end{abstract}

Compilation of a mouse strain-specific rDNA sequence. We previously sequenced a 625 -bp upstream region of the $r D N A$ from $B A L B / c$ mice (unpublished) and observed $>99 \%$ homology to a C57BL/6J sequence (GenBank NW_040290). To minimize mispairing of primers due to sequence variation between mouse strains, a full-length rDNA of C57BL/6J was compiled from two contigs (GenBank AC170254 and AF441733) by aligning with the assembled rDNA (GenBank BK000964) of the Swiss mouse strain. Primers were designed using Oligo 6 software (Molecular Biology Insights, Cascade, CO, USA) and the compiled C57BL/6 rDNA-homologous sequence.

RNA extraction and purification. Total RNA from A5 and E9 cells was extracted using the RNeasy kit (Qiagen, Germantown, MD, USA) and processed according to the manufacturer's protocol with a modification to extend the elution of RNA to $30 \mathrm{~min}$. The eluted RNA was subsequently treated with DNase to remove residual DNA, as described in the directions of the DNA-Free kit (Applied Biosystems, Foster City, CA, USA) and purified using Microcon 50 spin columns (Millipore, Bedford, MA, USA). RNA quantification was carried out using a spectrophotometer (Hewlett Packard, Mississauga, Ontario, Canada).

RT-PCR. The Superscript III enzyme was used to carry out RT at $50^{\circ} \mathrm{C}$ following the manufacturer's protocol (Invitrogen). Quantitative PCR was performed in the Chromo4 System (MJ Research, Waltham, MA, USA). PCR was carried out in a series of steps initiated by a 15 -min incubation at $95^{\circ} \mathrm{C}$. Subsequent three-step reactions were repeated for a total of 50 times, consisting of $94^{\circ} \mathrm{C}$ for $10 \mathrm{~s}, 55^{\circ} \mathrm{C}$ for $30 \mathrm{~s}$, and $72^{\circ} \mathrm{C}$ for $90 \mathrm{~s}$. Each PCR reaction contained $2 \mu \mathrm{l} \mathrm{CDNA}, 0.5 \mu \mathrm{M}$ primers, and $1 x$ SYBR Green PCR MIX (Qiagen) in $20 \mu$ l of solution. Samples from reactions omitting reverse transcriptase were used to verify that the reaction did not amplify residual DNA. Primers used for screening putative sense and antisense nc-rRNAs were chosen with similar computed melting temperatures (Supplementary Table S1). The probes and RT-PCR primers for the sense nc-rRNA enrichment analysis, sequence determination of the $5^{\prime}$ - and $3^{\prime}$-termini of the enriched sense nc-rRNAs, and test of ribozyme activities are listed in Supplementary Table S2. The transcripts of sense and antisense nc-rRNAs and pre-rRNAs containing the leader sequence and other $5^{\prime}$ ETS region were also quantified using real-time PCR after RT. RT primers were: $5^{\prime}$-GAGACAAACCTGGAACG-3' (for the two pre-rRNAs), $5^{\prime}$-ACC TATCTCCAGGTCCAATAGG-3' (for sense nc-rRNAs), and poly-T20 (for antisense nc-rRNAs). The corresponding PCR primers for each region were $5^{\prime}$-GTGGAGAGT CCCGAGTACTT-3'/5'-GGGGCAAGACAGTTACTGATA-3' (leader sequence), 5'-ACGTGTTTCACTTTGGTCGT-3'/5'-ACCTCGACGCTTACAAGAAA-3' (5' ETS),
5'-GCGGTTTTCTTTCATTGACC-3'/5'-ACCTATCTCCAGGTCCAATAGG-3' (sense nc-rRNAs), and $5^{\prime}$-GCGGTTTTCTTTCATTGACC-3'/5'-CCTTAAATCGAAAGGGT CTCTT- $3^{\prime}$ (antisense nc-rRNAs). The Gapdh was used as an internal control.

Enrichment of putative sense nc-rRNAs. A magnetic capturehybridization protocol, based on the hybridization of a biotinylated probe to the target transcript and capture of the hybrid with streptavidin-coated magnetic beads, was developed to enrich putative sense nc-rRNAs. The magnetic sphere and stand were purchased from Promega (PolyATtract Isolation System III, Madison, WI, USA), and several conditions were tested to maximize the isolation of sense ncrRNAs. Biotinylated probes (Supplementary Table S2) were designed to bind specifically to the $5^{\prime}$ regions of sense nc-rRNAs that are not present in the primary rRNA transcript. For the first condition, $100 \mu \mathrm{g}$ total RNAs were heat-denatured at $65^{\circ} \mathrm{C}$ for $5 \mathrm{~min}$ in the presence of probes only. The second condition included 30 oligonucleotides (Supplementary Table S1) in the heat denaturation step. The third condition was the same as the second, except inclusion of various concentrations of biotin in the $0.1 \times$ sodium chloride/sodium citrate wash buffer $(15 \mathrm{mM} \mathrm{NaCl}, 1.7 \mathrm{mM}$ $\mathrm{Na}_{3}$ citrate, $\mathrm{pH}$ 7.2). Duplicated RT and PCR were performed independently. The level of enrichment was expressed as a ratio to the $18 S$ rRNA and to Gapdh internal controls.

Determination of $\mathbf{5}^{\prime}$ - and $\mathbf{3}^{\prime}$-termini of sense nc-rRNAs. The sense nc-rRNAs enriched by the magnetic capture hybridization were phosphorylated at the $5^{\prime}$-end using T4 polynucleotide kinase and subsequently circularized by T4 RNA ligase 1 (New England BioLabs, Ipswich, MA, USA). Phosphorylation and circularization were carried out in a $50-\mu l$ reaction mixture containing the same buffer ( $50 \mathrm{mM}$ Tris- $\mathrm{HCl}, 10 \mathrm{mM} \mathrm{MgCl}_{2}, 1 \mathrm{mM}$ ATP, and $10 \mathrm{mM}$ dithiothreitol, $\mathrm{pH} \mathrm{7.8)}$ and an aliquot of the bead-enriched RNAs. The reaction was first incubated with $10 \mathrm{U}$ of T4 kinase for $30 \mathrm{~min}$ at $37^{\circ} \mathrm{C}$, followed by the addition of $20 \mathrm{U}$ of T4 ligase 1 and overnight incubation at $16^{\circ} \mathrm{C}$. Regions of the joint $5^{\prime}$ - and $3^{\prime}$-termini of circularized sense nc-rRNAs were PCR-amplified and determined by dideoxy DNA sequencing.

Test of ribozyme activities of nc-rRNAs. An aliquot of $3 \mu \mathrm{g}$ total RNA in $100 \mu$ liethyl pyrocarbonate (DEPC) water was processed through serial steps of heating at $65^{\circ} \mathrm{C}$ for $5 \mathrm{~min}$, cooling on ice for $5 \mathrm{~min}$, incubating for $1 \mathrm{~h}$ in a solution at $4{ }^{\circ} \mathrm{C}$, and purification by Microcon 30 before RT-PCR. For ribozyme characterization, 30 rRNA- or 6 Gapdh-specific complementary oligonucleotides (Supplementary Tables S1 and S2) were added before the $65-{ }^{\circ} \mathrm{C}$ heating step. DEPC water or $10 \mathrm{mM} \mathrm{MgCl}_{2}$ was introduced at the $1-\mathrm{h} 4{ }^{\circ} \mathrm{C}$ incubation step.

Amplicon deep sequencing. Initial PCRs were carried out using CDNAs, generated with RT primers (Supplementary Table S1) and total RNAs from A5 cells, and the same forward concatenated primer in combination with 26 reverse primers (Supplementary Table S3). Products of the 26 PCRs were resolved in DNA Bioanalyzer 2100 (Agilent Technologies, Santa Clara, CA, USA) and quantified. The concentrations of the $26 \mathrm{PCR}$ products were adjusted before pooling to have equal representation of individual products. The 454 GS FLX instrument and Titanium amplicon sequencing kit were used, as described in the manufacturer's protocol (Roche, Indianapolis, IN, USA). DNA sequencing was performed at $\times 500$ coverage.

Gel electrophoresis and dideoxy DNA sequencing. PCR products were separated on a $10 \%$ polyacrylamide gel (Invitrogen), stained with $0.5 \mu \mathrm{g} / \mathrm{ml}$ of ethidium bromide, and visualized using an Epi Chem II Darkroom Imaging System (UVP, Upland, CA, USA). Product sizes were estimated in reference to a 50-bp molecular weight marker. For DNA sequencing, PCR products were purified using the GFX purification columns (General Electric, Cleveland, OH, USA) and sequences were determined by conventional Sanger's dideoxy method.

Transfection. A5 and E9 cells at about $40-60 \%$ density in six-well plates were transfected with $50 \mathrm{nM}$ LNA-DNA gapmer (Sigma-Aldrich, St. Louis, MO, USA) using the RNAiFect transfection kit (Qiagen) according to the manufacturer's instruction. LNA1-AS (TACCtggaagtcataCCTG, upper cases denote LNAs), LNA1AS1 (TACCTggaagtcatACCTG), LNA2-AS (TAACActaaaggacACTAG), LNA2-AS1 (TAACActaaaggacACTAT), and LNA3-AS (AAAaagatccaaagctCCA) are complementary to sense nc-rRNAs, whereas LNA1-S (CAGGtatgacttccaGGTA), LNA2-S (TAAcactaaaggacacTAT), LNA2-S1 (TAAcactaaaggacacTAG), LNA3-S (TGGagctt ggatctTTT), and LNA4-S (TGTCCaagtgttcaTGCCA) are pairing with antisense nc-rRNAs. Cell images were taken utilizing a Zeiss Imaging System (Carl Zeiss 
Microlmaging, Inc., Thornwood, NY, USA). In-vitro synthesized nc-rRNAs ( $8 \mathrm{nM})$, transcribed from PCR templates and purified as previously described, ${ }^{28}$ were transfected into cells cultured in a 96-well plate using TransIT-mRNA Transfection kit (Mirus, Madison, WI, USA) according to the manufacturer's protocol.

Cell viability, apoptosis, and cell-cycle analyses. CellTiter $96 \mathrm{AQ}$ one solution cell proliferation kit (Promega) converting a tetrazolium compound to formazan for cell-viability measurement and Apo-BrdU (Phoenix Flow Systems, San Diego, CA, USA) for apoptosis as well as cell-cycle analyses were carried out according to manufacturer's instructions. Cells were cultured in a 96-well plate for CellTiter and in T-25 flasks for Apo-BrdU assays. Assessment of cell viability, cytotoxicity, and apoptosis from the same cell sample in 96-well plate was carried out using ApoTox-Glo Triplex Assay kit (Promega) following the manufacturer's direction. More than 10000 cells were collected after the Apo-BrdU staining using a Flow Cytometer. WinMDI 2.8 (http://www.cyto.purdue.edu/flowcyt/software/ Winmdi.htm) and Cylchred (http://www.facslab.toxikologie.uni-mainz.de/engl.\%20 Websites/Downloads-engl.jsp) software were used to calculate the fraction of apoptotic cells and three phases of cell cycle, respectively.

Statistical analysis. Nonparametric Mann-Whitney test was applied to compare treated and untreated samples in sense nc-rRNA enrichment experiment and ribozyme-like characterization experiments. For ribozyme-like characterization and changes of nc-rRNAs, pre-rRNAs, and cell populations (viability, cytotoxicity, apoptosis, and/or cell cycle) after LNA or synthetic nc-rRNAs treatments, unpaired Student's $t$-test was used. The differences between comparisons were considered significant if $P$-values were $<0.05$.

\section{Conflict of Interest}

The authors declare no conflict of interest.

Acknowledgements. We would like to thank Claudia Steward and Robin Steward at the Laboratory of Molecular Technology, SAIC-Frederick, for 454 amplicon deep sequencing and dideoxy DNA sequencing, Natalia Volfovsky at the Advanced Biomedical Computing Center, Information System Program, SAIC-Frederick, for bioinformatic analysis of deep-sequencing data, and Kathleen Noer and Guity Mohammadi at the Center for Cancer Research-Frederick Flow Cytometry Core for the flow analysis. We also appreciate colleague Bruce Shapiro for critical reading of the manuscript. This research was supported in part by the Intramural Research Program of the $\mathrm{NIH}$, National Cancer Institute, Center for Cancer Research.

1. Okazaki Y, Furuno M, Kasukawa T, Adachi J, Bono H, Kondo S et al. Analysis of the mouse transcriptome based on functional annotation of 60,770 full-length cDNAs. Nature 2002; 420: 563-573

2. Ota T, Suzuki Y, Nishikawa T, Otsuki T, Sugiyama T, Irie R et al. Complete sequencing and characterization of 21,243 full-length human cDNAs. Nat Genet 2004; 36: 40-45.

3. Frith MC, Pheasant M, Mattick JS. The amazing complexity of the human transcriptome. Eur J Hum Genet 2005; 13: 894-897.

4. Mattick JS, Makunin IV. Non-coding RNA. Hum Mol Genet 2006; 15: R17-R29.

5. Yelin R, Dahary D, Sorek R, Levanon EY, Goldstein O, Shoshan A et al. Widespread occurrence of antisense transcription in the human genome. Nat Biotechnol 2003; 21 379-386.

6. Chen J, Sun M, Kent WJ, Huang X, Xie H, Wang W et al. Over $20 \%$ of human transcripts might form sense-antisense pairs. Nucleic Acids Res 2004; 32: 4812-4820

7. Katayama S, Tomaru Y, Kasukawa T, Waki K, Nakanishi M, Nakamura M et al. Antisense transcription in the mammalian transcriptome. Science 2005; 309: 1564-1566.

8. Faghihi MA, Wahlestedt $\mathrm{C}$. Regulatory roles of natural antisense transcripts. Nature Rev Mol Cell Biol 2009; 10: 637-643.

9. Martianov I, Ramadass A, Serra Barros AS, Chow A, Akoulitchev A. Repression of the dihydrofolate reductase gene by a non-coding interfering transcript. Nature 2007; 445 $666-670$.

10. Martens JA, Laprade L, Winston F. Intergenic transcription is required to repress the Saccharomyces cerevisiae SER3 gene. Nature 2004; 429: 571-574.

11. Shiao YH, Lupascu ST, Gu YD, Kasprzak W, Hwang CJ, Fields JR et al. An intergenic noncoding rRNA correlated with expression of the rRNA and frequency of an rRNA single nucleotide polymorphism in lung cancer cells. PLoS One 2009; 4: e7505.

12. Henderson S, Sollner-Webb B. A transcriptional terminator is a novel element of the promoter of the mouse ribosomal RNA gene. Cell 1986; 47: 891-900.

13. Grummt I, Kuhn A. A novel promoter in the mouse rDNA spacer is active in vivo and in vitro. EMBO J 1987; 6: 3487-3492.

14. Mayer C, Schmitz K-M, Li J, Grummt I, Santoro R. Intergenic transcripts regulate the epigenetic state of rRNA genes. Mol Cell 2006; 22: 351-361.

15. Gurney T. Characterization of mouse $45 \mathrm{~S}$ ribosomal RNA subspecies suggests that the first processing cleavage occurs $600+/-100$ nucleotides from the $5^{\prime}$-end and the second $500+/$ - nucleotides from the 3 '-end of a $13.9 \mathrm{~kb}$ precursor. Nucleic Acids Res 1985; 13: 4905-4919.

16. Eichler DC, Craig N. Processing of eukaryotic ribosomal RNA. Prog Nucleic Acid Res 1994; 49: $197-239$.

17. Ruggero D, Pandolfi PP. Does the ribosome translate cancer? Nature Rev Cancer 2003; $\mathbf{3}$ : 179-192.

18. White RJ. RNA polymerases I and III, non-coding RNAs and cancer. Trends Genet 2008; 24: $622-629$.

19. Sithanandam G, Smith GT, Fields JR, Fornwald LW, Anderson LM. Alternate paths from epidermal growth factor receptor to Akt in malignant versus nontransformed lung epithelial cells: Erb3 versus Gab1. Am J Respir Cell Mol Biol 2005; 33: 490-499.

20. Parlato R, Kreiner G, Erdmann G, Rieker C, Stotz S, Savenkova E et al. Activation of an endogenous suicide response after perturbation of rRNA synthesis leads to neurodegeneration in mice. J Neurosci 2008; 28: 12759-12764.

21. Bierhoff H, Schmitz K, Maass F, Ye J, Grummt I. Noncoding transcripts in sense and antisense orientation regulate the epigenetic state of ribosomal RNA genes. Cold Spring Harb Symp Quant Biol 2010; 75: 357-364.

22. Eickbush DG, Eickbush TH. R2 retrotransposons encode a self-cleaving ribozyme for processing from an rRNA cotranscript. Mol Cell Biol 2010; 30: 3142-3150.

23. Lonnberg T, Lonnberg H. Chemical models for ribozyme action. Curr Opin Chem Biol 2005; 9: 665-673.

24. Vourekas A, Stamatopoulou V, Toumpeki C, Tsitlaidou M. Insights into functional modulation of catalytic RNA activity. IUBMB Life 2008; 60: 669-683.

25. Matsui K, Nishizawa M, Ozaki T, Kimura T, Hashimoto I, Yamada M et al. Natural antisense transcript stabilizes inducible nitric oxide synthase messenger RNA in rat hepatocytes. Hepatology 2008; 47: 686-697.

26. Faghihi MA, Modarresi F, Khalil AM, Wood DE, Sahagan BG, Morgan TE et al. Expression of a noncoding RNA is elevated in Alzheimer's disease and drives rapid feed-forward regulation of beta-secretase. Nat Med 2008; 14: 723-730.

27. Gagnon-Kugler T, Langlois F, Stefanovsky V, Lessard F, Moss T. Loss of human ribosomal gene $\mathrm{CpG}$ methylation enhances cryptic RNA polymerase II transcription and disrupts ribosomal RNA processing. Mol Cell 2009; 35: 414-425.

28. Hwang CJ, Fields JR, Shiao YH. Noncoding rRNA-mediated preferential killing in cancer cells is enhanced by suppression of autophagy in nontransformed counterpart. Cell Death Dis 2011; 2: e239.

29. Cohen MB, Glazer RI. Cytotoxicity and the inhibition of ribosomal RNA processing in human colon carcinoma cells. Mol Pharmacol 1985; 27: 308-313.

30. Ghoshal K, Jacob ST. Specific inhibition of pre-ribosomal RNA processing in extracts from the lymphosarcoma cells treated with 5-fluorouracil. Cancer Res 1994; 54: 632-636

31. Burger K, Muhl B, Harasim T, Rohrmoser M, Malamoussi A, Orban M et al. Chemotherapeutic drugs inhibit ribosome biogenesis at various levels. $\mathrm{J}$ Biol Chem 2010; 285: 12416-12425.

32. Yen L, Magnier M, Weissleder R, Stockwell BR, Mulligan RC. Identification of inhibitors of ribozyme self-cleavage in mammalian cells via high-throughput screening of chemical libraries. RNA 2006; 12: 797-806.

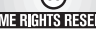

Cell Death and Disease is an open-access journal published by Nature Publishing Group. This work is licensed under the Creative Commons Attribution-Noncommercial-No Derivative Works 3.0 Unported License. To view a copy of this license, visit http://creativecommons.org/licenses/by-nc-nd/3.0/

\section{Supplementary Information accompanies the paper on Cell Death and Disease website (http://www.nature.com/cddis)}

